\title{
On Ŝostak's fuzzy contra S-pre-continuous mappings
}

\author{
B. Vijayalakshmi ${ }^{1 *}$, M. Sujatha ${ }^{2}$, M. Angayarkanni ${ }^{3}$, and A. Vadivel ${ }^{4}$
}

\begin{abstract}
Fuzzy contra S-pre-continuous, fuzzy contra S-preopen, fuzzy contra S-preclosed mappings and fuzzy contra S-pre-homeomorphism with properties are introduced and studied in Šostak's sense. Also a comparison between these new types of functions are established by giving examples.

Keywords

fuzzy contra S-pre-continuous, fuzzy contra S-preopen, fuzzy contra S-preclosed.

AMS Subject Classification

54A40, 54C05, 03E72.

${ }^{1}$ Department of Mathematics, Government Arts College, Chidambaram -608102, India.

${ }^{2}$ Department of Mathematics, Padmavani Arts and Science College for Women, Salem- 636 011, India.

${ }^{3}$ Department of Mathematics, Kandaswamy Kandara's college, P-Velur-638182, India.

${ }^{4}$ Department of Mathematics, Annamalai University, Annamalai Nagar - 608002, India.

*Corresponding author: ${ }^{\text {* }}$ mathvijaya2006au@gmail.com; ${ }^{4}$ avmaths@gmail.com

Article History: Received 24 November 2018; Accepted 09 April 2019

(C)2019 MJM.
\end{abstract}

\section{Contents}

1 Introduction and Preliminaries .427

2 Fuzzy Contra S-pre-continuous Mappings ...... 428

3 Conclusion ............................ 431

References ..............................431

\section{Introduction and Preliminaries}

After the introduction of fuzzy sets by Zadeh [29], Chang[5] was the one who initiated the idea of a fuzzy topology in a set $X$, in which a collection, $T$, of open sets of fuzzy subsets of $X$. He also defined new type of fuzzy topology. In [7, 10], the authors defined the idea of gradation of openness of fuzzy subsets of $X$. They also used the concept of fuzzy topology in the sense of Chang. In 1980 in [11],the basic idea of topology is itself fuzzy. As in 1985 by the customized work of Kubiak [14] and $\breve{S}$ ostak [21] in Lattice $L=I$ was initiated in 1995 by [12] and further by [15] in 1997, in that topology was used to explain the two membership latices namely $L^{X}$ to $M$, where $L$ and $M$ are appropriate lattices. With all the basic ideas of topologies the basic lattices are fixed in $[14,21]$ and another significant generalizations of lattices $L$ varies from space to space [13] is also represented. Using the developments of [11-15, 20-24], the notion of a fuzzy topology as a fuzzy subset tof powerset is independently consider with the base work of [11, 14, 21]. At present authors Chattopadhyay [7] et.al., Ramadan [19] and Ying [28] are also worked in this concept.

In this article, fuzzy contra S-pre-continuous, fuzzy contra S-preopen, fuzzy contra S-preclosed mappings and fuzzy contra S-pre-homeomorphism with properties are introduced and studied in Šostak's sense. Also a comparison between these new types of functions are established by giving examples.

$X, Y$ etc., denotes the non empty sets, $I=[0,1]$ and $I_{0}=(0,1]$. In 1992 Ramadan [19] given the definition of smooth topological spaces (in short, sts) and the operators $C_{\tau}: I^{X} \times I_{0} \rightarrow I^{X}$ as $C_{\tau}(\lambda, r)=\bigwedge\left\{\mu \in I^{X}: \lambda \leq \mu, \tau(\overline{1}-\right.$ $\mu) \geq r\}$. and $I_{\tau}: I^{X} \times I_{0} \rightarrow I^{X}$ as $I_{\tau}(\lambda, r)=\bigvee\left\{\mu \in I^{X}: \lambda \geq\right.$ $\mu, \tau(\mu) \geq r\}$. Kim et. al.[16] discussed in a sts $(X, \tau)$, $\forall r \in I_{0} . \lambda$ is called $r$-fuzzy strongly semiopen (resp. $r$-fuzzy semiopen $\& r$-fuzzy preopen) ( $r$-fsso (resp. $r$-fso $\& r$-fpo) for short) iff $\lambda \leq I_{\tau}\left(C_{\tau}\left(I_{\tau}(\lambda, r), r\right), r\right)$ (resp. $\lambda \leq C_{\tau}\left(I_{\tau}(\lambda, r), r\right)$ $\left.\& \lambda \leq I_{\tau}\left(C_{\tau}(\lambda, r), r\right)\right)$. The respective compliment sets are closed set.

The operators $r$-fuzzy strongly semi-interior (resp. $r$-fuzzy pre-interior, $r$-fuzzy strongly semi-closure and $r$-fuzzy preclosure) of $\lambda$, denoted by $S S I_{\tau}(\lambda, r)$ (resp. $P I_{\tau}(\lambda, r), S S C_{\tau}$ $(\lambda, r)$ and $\left.P C_{\tau}(\lambda, r)\right)$ as $S S I_{\tau}(\lambda, r)\left(\operatorname{resp} . P I_{\tau}(\lambda, r)\right)=$ $\bigvee\left\{\mu \in I^{X}: \mu \leq \lambda, \mu\right.$ is $r$-fsso (resp. $r$-fpo) $\}$ (resp. $S S C_{\tau}$ $(\lambda, r)\left(\right.$ resp. $\left.P C_{\tau}(\lambda, r)\right)=\bigwedge\left\{\mu \in I^{X}: \mu \geq \lambda, \mu\right.$ is $r$-fssc $($ resp . $r$-fpc) $\})$. 
$f:(X, \tau) \rightarrow(Y, \eta)$ is fuzzy continuous (FCts for short) iff $\eta(\mu) \leq \tau\left(f^{-1}(\mu)\right)$ for each $\mu \in I^{Y} . f:(X, \tau) \rightarrow(Y, \eta)$ is fuzzy semi continuous, (resp. fuzzy irresolute and fuzzy S-irresolute continuous) (FSCts (resp. FI and FS-ICts) for short) iff $f^{-1}(\mu)$ is $r$-fso (resp. $r$-fso and $r$-fsso) set of $X$ for each $\eta(\mu) \geq r$, (resp. $\mu$ is $r$-fso and $r$-fsso )set of $Y r \in I_{0}$. $f:(X, \tau) \rightarrow(Y, \eta)$ is fuzzy open(resp. fuzzy closed ) (FO (resp. FC) for short) iff $\tau(\lambda) \leq \eta(f(\lambda))$ (resp. $\tau(\overline{1}-\lambda) \leq$ $\eta(\overline{1}-f(\lambda)))$ for each $\lambda \in I^{X} . f:(X, \tau) \rightarrow(Y, \eta)$ is fuzzy $S$-irresolute open (resp. fuzzy strongly semi-open and fuzzy strongly semi-closed) (FS-IO (resp. FsSO and FsSCC) for short) iff $f(\mu)$ is $r$-fsso (resp. $r$-fsso and $r$-fssc) set of $Y$ for each $r$-fsso (resp. $\tau(\mu) \geq r \& \operatorname{tau}(\overline{1}-\mu) \geq r$, ) set $r \in I_{0}$.

$f:(X, \tau) \rightarrow(Y, \eta)$ is fuzzy irresolute preopen (resp. fuzzy irresolute preclosed) [25] iff $f(\mu)$ is $r$-fpo (resp. $r$-fpc) set of $X$, for each $r$-fpo (resp. $r$-fpc) set $\mu \in I^{Y}, r \in I_{0}$.

$f:(X, \tau) \rightarrow(Y, \eta)$ is fuzzy contra continuous [2] (resp. fuzzy contra pre-continuous [8]) (FcCts (resp. FcPCts) for short) if for each $\mu \in I^{Y}$ (resp. $\left.\eta(\mu) \geq r\right)$ we have $\tau(\overline{1}-$ $\left.f^{-1}(\mu)\right) \geq \eta(\mu)$ (resp. $f^{-1}(\mu)$ is $r$-fpc). $f:(X, \tau) \rightarrow(Y, \eta)$ is fuzzy contra open(resp. fuzzy contra closed) ( $\mathrm{FcO}$ (resp. $\mathrm{FcC}$ ) for short) if for each $\lambda \in I^{X}$ we have $\eta(\overline{1}-f(\lambda)) \geq \tau(\lambda)$ (resp. $\eta(f(\lambda)) \geq \tau(\overline{1}-\lambda)$ ). $f:(X, \tau) \rightarrow(Y, \eta)$ is fuzzy contra S-irresolute continuous [26] (resp. fuzzy contra semiS-irresolute continuous [27]) (FcS-ICts (resp. FcSS-ICts) for short)iff $f^{-1}(\mu)$ is $r$-fssc (resp. $r$-fsc) set of $X$ for each $r$-fsso set $\mu \in I^{Y}, r \in I_{0}$.

The pair $(X, \tau)$ is called a smooth topological space (or) Šostak Fuzzy topological space (or) fuzzy topological spaces (in short, sts, sfts, fts).

\section{Fuzzy Contra S-pre-continuous Mappings}

Definition 2.1. A function $f:(X, \tau) \rightarrow(Y, \eta)$ is called

(1) fuzzy contra S-pre-continuous ( $F$ cS-PCts, for short) iff $f^{-1}(\mu)$ is $r$-fpc set of $X \forall r$-fsso set $\mu \in I^{Y}, r \in I_{0}$.

(2) fuzzy contra S-preopen (resp. fuzzy contra S-preclosed) (FcS-PO (resp. FcS-PC), for short) iff $f(\mu)$ is $r$-fpc (resp. $r$-fpo) set of $Y \forall r$-fsso (resp. $r$-fssc) set $\mu \in I^{X}$, $r \in I_{0}$.

(3) fuzzy contra S-pre homeomorphism ( $F c S-P H$, for short) iff $f$ is bijective and both of $f \& f^{-1}$ are fuzzy contra S-pre-continuous.

Based on the definitions,

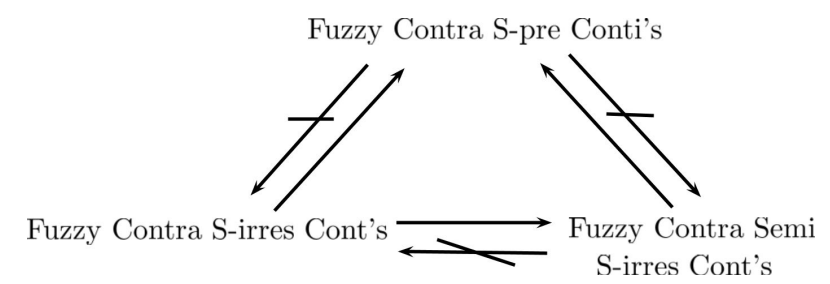

Remark 2.2. The reverse implications not true in general, which is shown in the succeeding example.

Example 2.3. Consider the fts's $(X, \tau) \&(Y, \eta)$ with $X=Y=$ $\{x, y, z\}$ and

$\tau(\lambda)= \begin{cases}1, & \text { if } \lambda=\overline{0} \text { or } \overline{1} \\ \frac{1}{2}, & \text { if } \lambda=\alpha_{1} \\ 0, & \text { otherwise }\end{cases}$

$\eta(\mu)= \begin{cases}1, & \text { if } \mu=\overline{0} \text { or } \overline{1} \\ \frac{1}{2}, & \text { if } \mu=\alpha_{2} \\ 0, & \text { otherwise }\end{cases}$

where $\alpha_{1}(x)=0.2, \alpha_{1}(y)=0.1, \alpha_{1}(z)=0.5 ; \quad \alpha_{2}(x)=$ $0.7, \alpha_{2}(y)=0.9, \quad \alpha_{2}(z)=0.6 ; \quad \alpha_{3}(x)=0.8, \alpha_{3}(y)=1$, $\alpha_{3}(z)=0.7$.

Then the identity function $F: X \rightarrow Y$ is

(i) FCS-PCts but not FCS-ICts because $\alpha_{3}$ is $\frac{1}{2}$-fsso in $(Y, \eta)$ but $f^{-1}\left(\alpha_{3}\right)=\alpha_{3}$ is not $\frac{1}{2}$-fssc in $(X, \tau)$.

(ii) FcS-PCts but not FcSS-ICts continuous because $\alpha_{3}$ is $\frac{1}{2}$-fsso in $(Y, \eta)$ but $f^{-1}\left(\alpha_{3}\right)=\alpha_{3}$ is not $\frac{1}{2}$-fsc in $(X, \tau)$.

Example 2.4. Consider the fts's $(X, \tau) \&(Y, \eta)$ with $X=Y=$ $\{x, y, z\}$ and

$\tau(\lambda)= \begin{cases}1, & \text { if } \lambda=\overline{0} \text { or } \overline{1} \\ \frac{1}{2}, & \text { if } \lambda=\alpha_{1} \\ 0, & \text { otherwise }\end{cases}$

$\eta(\mu)= \begin{cases}1, & \text { if } \mu=\overline{0} \text { or } \overline{1} \\ \frac{1}{2}, & \text { if } \mu=\alpha_{2} \\ 0, & \text { otherwise }\end{cases}$

where $\alpha_{1}(x)=0.2, \alpha_{1}(y)=0.1, \alpha_{1}(z)=0 ; \quad \alpha_{2}(x)=0.5$, $\alpha_{2}(y)=0.7, \alpha_{2}(z)=0$.

Then the identity function $f: X \rightarrow Y$ is FcSS-ICts but not FCS-ICts because $\alpha_{2}$ is $\frac{1}{2}-f$ sso in $(Y, \eta)$ but $f^{-1}\left(\alpha_{2}\right)=\alpha_{2}$ is not $\frac{1}{2}-$ fssc in $(X, \tau)$.

Theorem 2.5. For a mapping $f:\left(X, \tau_{1}\right) \rightarrow\left(Y, \tau_{2}\right)$, the succeeding statements are equivalent.

(1) $f$ is FcS-PCts.

(2) $\forall r$-fssc $v \in I^{Y}, f^{-1}(v)$ is $r$-fpo.

(3) $f\left(I_{\tau_{1}}\left(C_{\tau_{1}}(\mu, r), r\right)\right) \geq \operatorname{SSC}_{\tau_{2}}(f(\mu), r)$, for each $\mu \in I^{X}$ and $r \in I_{0}$.

(4) $I_{\tau_{1}}\left(C_{\tau_{1}}\left(f^{-1}(v), r\right), r\right) \geq f^{-1}\left(S S C_{\tau_{2}}(v, r)\right.$, for each $v \in I^{Y}$ and $r \in I_{0}$.

(5) $f^{-1}(v) \geq C_{\tau_{1}}\left(I_{\tau_{1}}\left(f^{-1}(v), r\right), r\right)$, for each $r$-fsso set $v \in I^{Y}$ and $r \in I_{0}$. 
Proof. Using the fact $f^{-1}(\overline{1}-v)=\overline{1}-f^{-1}(v),(1) \Rightarrow(2)$, can be obtained directly.

(2) $\Rightarrow(3)$ : Suppose $\exists \mu \in I^{Y} \& r \in I_{0} \ni$

$$
f\left(I_{\tau_{1}}\left(C_{\tau_{1}}(\mu, r), r\right)\right) \nsupseteq S S C_{\tau_{2}}(f(\mu), r) .
$$

There exists $y \in Y \& t \in(0,1) \ni f\left(I_{\tau_{1}}\left(C_{\tau_{1}}(\mu, r), r\right)\right)(y)<$ $t<S S C_{\tau_{2}}(f(\mu), r)(y)$.

If $f^{-1}(\{y\})=\phi$, it contradicts $f\left(I_{\tau_{1}}\left(C_{\tau_{1}}(\mu, r), r\right)\right)(y)=0$.

If $f^{-1}(\{y\}) \neq \phi, \exists x \in f^{-1}(\{y\})$ such that

$$
\begin{aligned}
f\left(I_{\tau_{1}}\left(C_{\tau_{1}}(\mu, r), r\right)\right)(y) & \leq I \tau_{1}\left(C_{\tau_{1}}(\mu, r), r\right)(x) \\
& <t \\
& <S S C_{\tau_{2}}(f(\mu), r)(f(x))
\end{aligned}
$$

Since $\operatorname{SSC}_{\tau_{2}}(f(\mu), r)(f(x))>t, \exists r$-fssc $v \in I^{Y}$ with $f(\mu) \geq$ $v$ such that

$$
\operatorname{SSC}_{\tau_{2}}(f(\mu), r)(f(x)) \geq v(f(x))>t .
$$

And $f(\mu) \geq v$ then $\mu \geq f^{-1}(v)$.

(2) $\Rightarrow f^{-1}(v)$ is $r$-fpo. Hence

$$
I_{\tau_{1}}\left(C_{\tau_{1}}(\mu, r), r\right)(x) \geq f^{-1}(v)(x)=v(f(x))>t .
$$

It contradicts equation (2.1).

Thus $f\left(I_{\tau_{1}}\left(C_{\tau_{1}}(\mu, r), r\right)>S S C_{\tau_{2}}(f(\mu), r)\right.$.

(3) $\Rightarrow(4): \forall v \in I^{Y}, r \in I_{0}$, put $\mu=f^{-1}(v)$. From (3),

$$
\begin{aligned}
f\left(I_{\tau_{1}}\left(C_{\tau_{1}}\left(f^{-1}(v), r\right), r\right)\right) & \geq \operatorname{SSC}_{\tau_{2}}\left(f\left(f^{-1}(v)\right), r\right) \\
& \geq \operatorname{SSC}_{\tau_{2}}(v, r)
\end{aligned}
$$

It implies

$$
\begin{aligned}
I_{\tau_{1}}\left(C_{\tau_{1}}\left(f^{-1}(v), r\right), r\right) & \geq f^{-1}\left(I_{\tau_{1}}\left(C_{\tau_{1}}\left(f^{-1}(v), r\right), r\right)\right) \\
& \geq f^{-1}\left(S S C_{\tau_{2}}(v, r)\right) .
\end{aligned}
$$

Thus proved (4).

(4) $\Rightarrow(3)$ : Let $\mu \in I^{X} \& r \in I_{0}$. By (4),

$$
\begin{aligned}
I_{\tau_{1}}\left(C_{\tau_{1}}(\mu, r), r\right) & \geq I_{\tau_{1}}\left(C_{\tau_{1}}\left(f^{-1}(f(\mu)), r\right), r\right) \\
& \geq f^{-1}\left(S S C_{\tau_{2}}(f(\mu), r)\right)
\end{aligned}
$$

and hence $f\left(I_{\tau_{1}}\left(C_{\tau_{1}}(\mu, r), r\right) \geq \operatorname{SSC}_{\tau 2}(f(\mu), r)\right.$.

Thus (3) is proved.

(3) $\Leftrightarrow(1)$ : Take $v \in I^{Y}$ be a $r$-fsso set.

Since $f^{-1}(\overline{1}-v)=\overline{1}-f^{-1}(v)$, by (3),

$$
\begin{aligned}
f\left(I _ { \tau _ { 1 } } \left(C _ { \tau _ { 1 } } \left(f^{-1}(\overline{1}-v),\right.\right.\right. & r), r)) \geq S S C_{\tau_{2}}\left(f f^{-1}(\overline{1}-v), r\right) \\
& \geq S S C_{\tau_{2}}(\overline{1}-v, r) \\
& =\overline{1}-S_{S I} I_{\tau_{2}}(v, r) \\
& \geq \overline{1}-v
\end{aligned}
$$

and hence $\overline{1}-C_{\tau_{1}}\left(I_{\tau_{1}}\left(f^{-1}(v), r\right), r\right)$

$$
\begin{aligned}
& =I_{\tau_{1}}\left(C_{\tau_{1}}\left(\overline{1}-f^{-1}(v), r\right), r\right) \\
& =I_{\tau_{1}}\left(C_{\tau_{1}}\left(f^{-1}(\overline{1}-v), r\right), r\right) \\
& \geq f^{-1} f\left(I_{\tau_{1}}\left(C_{\tau_{1}}\left(f^{-1}(\overline{1}-v), r\right), r\right)\right) \\
& \geq f^{-1}(\overline{1}-v) \\
& \geq \overline{1}-f^{-1}(v)
\end{aligned}
$$

Therefore, we obtain $f^{-1}(v) \geq C_{\tau_{1}}\left(I_{\tau_{1}}\left(f^{-1}(v), r\right), r\right)$

$\Rightarrow f^{-1}(v)$ is $r$-fpc set in $X$.

Hence the mapping $f$ is $F c S-P C t s$.

Theorem 2.6. Let $f:(X, \tau) \rightarrow(Y, \eta)$ be a bijective mapping. Then (1) \& (2) are equivalent.

(1) $f$ is FcS-PCts.

(2) $\operatorname{SSI}_{\eta}(f(\mu), r) \geq f\left(C_{\tau}\left(I_{\tau}(\mu, r), r\right)\right), \forall \mu \in I^{X}$ and $r \in$ $I_{0}$.

Proof. (1) $\Rightarrow(2)$ : Let $f$ be a FcS-PCts mapping and $\mu \in I^{X}$. Then $f^{-1}\left(\operatorname{SSI}_{\eta}(f(\mu), r)\right)$ is $r$-fpc set in $X$.

Since $f$ is $1-1$, and by Theorem $2.5, f^{-1}\left(\operatorname{SSI}_{\eta}(v, r)\right) \geq$ $C_{\tau}\left(I_{\tau}\left(f^{-1}(v), r\right), r\right)$. Let $v=f(\mu)$. Then,

$$
\begin{aligned}
f^{-1}\left(\operatorname{SSI}_{\eta}(f(\mu), r)\right) & \geq C_{\tau}\left(I_{\tau}\left(f^{-1}(f(\mu), r), r\right)\right. \\
& =C_{\tau}\left(I_{\tau}(\mu, r), r\right) .
\end{aligned}
$$

But, $f$ is onto, so

$$
\begin{aligned}
\operatorname{SSI}_{\eta}(f(\mu), r) & =f f^{-1}\left(\operatorname{SSI}_{\eta}(f(\mu), r)\right. \\
& \geq f\left(C_{\tau}\left(I_{\tau}(\mu, r), r\right)\right) .
\end{aligned}
$$

Thus (2) is proved.

(2) $\Rightarrow(1)$ : Consider $v \in I^{Y}$ be a $r$-fsso set. Using the Theorem in [16], $v=\operatorname{SSI}_{\eta}(v, r)$. By (2),

$$
\begin{aligned}
f\left(C_{\tau}\left(I_{\tau}\left(f^{-1}(v), r\right), r\right)\right) & =\operatorname{SSI}_{\eta}\left(f\left(f^{-1}(v), r\right)\right) \\
& =\operatorname{SSI}_{\eta}(v, r) \\
& =v
\end{aligned}
$$

and

$$
\begin{aligned}
C_{\tau}\left(I_{\tau}\left(f^{-1}(v), r\right), r\right) & =f^{-1} f\left(C_{\tau}\left(I_{\tau}\left(f^{-1}(v), r\right), r\right)\right) \\
& \leq f^{-1}(v) .
\end{aligned}
$$

Hence, $C_{\tau}\left(I_{\tau}\left(f^{-1}(v), r\right), r\right) \leq f^{-1}(v) \Rightarrow f^{-1}(v)$ is $r$-fpc. Thus (1).

Theorem 2.7. For a mapping $f:(X, \tau) \rightarrow(Y, \eta)$, the statements

(1) $f$ is FcS-PO.

(2) $f\left(\operatorname{SSI}_{\tau}(\mu, r)\right) \leq C_{\eta}\left(I_{\eta}(f(\mu), r), r\right), \forall \mu \in I^{X} \& r \in I_{0}$.

(3) $\operatorname{SSI}_{\tau}\left(f^{-1}(v), r\right) \leq f^{-1}\left(C_{\eta}\left(I_{\eta}(v, r), r\right)\right), \forall v \in I^{Y} \& r \in$ $I_{0}$.

(4) For any $r$-fssc $v \in I^{Y}$ with $f^{-1}(v) \leq \mu, \exists r$-fpo $\rho \in I^{Y}$ with $v \leq \rho \ni f^{-1}(\rho) \leq \mu$.

are equivalent. 
Proof. (1) $\Rightarrow(2)$ : Let $f$ be FcS-PO, $\forall \mu \in I^{X}$, since, $\operatorname{SSI}_{\tau}(\mu, r)$ $\leq \mu$ from Theorem in [16],

$$
f\left(\operatorname{SSI}_{\tau}(\mu, r)\right) \leq f(\mu) .
$$

From (1), $f\left(\operatorname{SSI}_{\tau}(\mu, r)\right)$ is $r$-fpc. Thus $f\left(\operatorname{SSI}_{\tau}(\mu, r)\right) \leq$ $C_{\eta}\left(I_{\eta}(f(\mu), r), r\right)$. Thus proved (2).

(2) $\Rightarrow(3): \forall v \in I^{Y}, r \in I_{0}$ let $\mu=f^{-1}(v)$ from (2). Then,

$$
\begin{aligned}
f\left(\operatorname{SSI}_{\tau}\left(f^{-1}(v), r\right)\right) & \leq C_{\eta}\left(I_{\eta}\left(f\left(f^{-1}(v)\right), r\right), r\right) \\
& \leq C_{\eta}\left(I_{\eta}(v, r), r\right) .
\end{aligned}
$$

It implies $\operatorname{SSI}_{\tau}\left(f^{-1}(v), r\right) \leq f^{-1}\left(C_{\eta}\left(I_{\eta}(v, r), r\right)\right)$.

(3) $\Rightarrow(4)$ : Take a $r$-fssc set $\mu \in I^{X} \ni f^{-1}(v) \leq \mu$. Since $\overline{1}-\mu \leq f^{-1}(\overline{1}-v)$ and $\operatorname{SSI}_{\tau}(\overline{1}-\mu, r)=\overline{1}-\mu$.

$$
\operatorname{SSI}_{\tau}(\overline{1}-\mu, r)=\overline{1}-\mu \leq \operatorname{SSI}_{\tau}\left(f^{-1}(\overline{1}-v), r\right) .
$$

(3) implies,

$$
\overline{1}-\mu \leq \operatorname{SSI}_{\tau}\left(f^{-1}(\overline{1}-v), r\right) \leq f^{-1}\left(C_{\eta}\left(I_{\eta}(\overline{1}-v, r), r\right)\right) .
$$

It implies

$$
\begin{aligned}
\mu & \geq \overline{1}-f^{-1}\left(C_{\eta}\left(I_{\eta}(\overline{1}-v, r), r\right)\right) \\
& =f^{-1}\left(\overline{1}-C_{\eta}\left(I_{\eta}(\overline{1}-v, r), r\right)\right) \\
\mu & \geq f^{-1}\left(I_{\eta}\left(C_{\eta}(v, r), r\right)\right)
\end{aligned}
$$

Thus a $r$-fpo set $v \in I^{Y}$ with $v \leq I_{\eta}\left(C_{\eta}(v, r), r\right) \ni$

$$
f^{-1}\left(I_{\eta}\left(C_{\eta}(v, r), r\right)\right) \leq \mu
$$

exists. Thus (4) is proved.

(4) $\Rightarrow(1)$ : Take a $r$-fsso set $\omega \in I^{X}$. Now, we have to prove, $f(\omega) \in I^{Y}$ is $r$-fpc. Let $v=\overline{1}-f(\omega) \& \mu=\overline{1}-\omega \ni \mu$ is $r$-fssc. Therefore

$f^{-1}(v)=f^{-1}(\overline{1}-f(\omega))=\overline{1}-f^{-1}(f(\omega)) \leq \overline{1}-\omega=\mu$ $\Rightarrow f^{-1}(v) \leq \mu$.

(4) implies, a $r$-fpo set $\rho$ with $v \leq \rho \ni f^{-1}(\rho) \leq \mu=$ $\overline{1}-\omega$ exists. It implies

$$
\omega \leq \overline{1}-f^{-1}(\rho)=f^{-1}(\overline{1}-\rho) .
$$

Thus,

$$
f(\omega) \leq f\left(f^{-1}(\overline{1}-\rho)\right) \leq \overline{1}-\rho
$$

On the other hand, since $v \leq \rho$,

$$
f(\omega)=\overline{1}-v \geq \overline{1}-\rho
$$

Hence from equations (2.2) and (2.3), we get, $f(\omega)=\overline{1}-\rho$.

(i.e) $f(\omega)$ is $r$-fpc. Thus (1).

Theorem 2.8. For a mapping $f:(X, \tau) \rightarrow(Y, \eta)$, the statements

(1) $f$ is $F c S-P C$
(2) $f\left(\operatorname{SSC}_{\tau}(\mu, r)\right) \geq I_{\eta}\left(C_{\eta}(f(\mu), r), r\right) \forall \mu \in I^{X}$ and $r \in$ $I_{0}$.

(3) $\operatorname{SSC}_{\tau}\left(f^{-1}(v), r\right) \geq f^{-1}\left(I_{\eta}\left(C_{\eta}(v, r), r\right)\right), \forall v \in I^{Y}$ and $r \in I_{0}$.

(4) For any $v \in I^{Y} \& r$-fsso $\mu \in I^{X}$ with $f^{-1}(v) \leq \mu, \exists r$ fpc, $\rho \in I^{Y}$ with $v \geq \rho \ni f^{-1}(\rho) \geq \mu$.

are equivalent.

Proof. (1) $\Rightarrow(2)$ : Let $f$ be FcS-PC. For each $\mu \in I^{X}$ and $r \in I_{0}$, since $\operatorname{SSC}_{\tau}(\mu, r) \geq \mu$ from Theorem ??(5), Then

$$
f\left(\operatorname{SSC}_{\tau}(\mu, r)\right) \geq f(\mu) .
$$

From (1), $f\left(\operatorname{SSC}_{\tau}(\mu, r)\right)$ is $r$-fpo. Therefore $f\left(\operatorname{SSC}_{\tau}(\mu, r)\right) \geq I_{\eta}\left(C_{\eta}(f(\mu), r), r\right)$. Thus (2) is proved.

(2) $\Rightarrow(3): \forall v \in I^{Y}, r \in I_{0}$ Take $\mu=f^{-1}(v)$ in (2) implies that,

$$
\begin{aligned}
f\left(\operatorname{SSC}_{\tau}\left(f^{-1}(v), r\right)\right) & \geq I_{\eta}\left(C_{\eta}\left(f\left(f^{-1}(v)\right), r\right), r\right) \\
& \geq I_{\eta}\left(C_{\eta}(v, r), r\right) .
\end{aligned}
$$

It implies $\operatorname{SSC}_{\tau}\left(f^{-1}(v), r\right) \geq f^{-1}\left(I_{\eta}\left(C_{\eta}(v, r), r\right)\right)$.

Thus proved (3) .

(3) $\Rightarrow(4)$ : Let $\mu$ be $r$-fsso set of $X$ such that $f^{-1}(v) \geq \mu$.

Since $\overline{1}-\mu \geq f^{-1}(\overline{1}-v)$ and $\operatorname{SSC}_{\tau}(\overline{1}-\mu, r)=\overline{1}-\mu$

$\operatorname{SSC}_{\tau}(\overline{1}-\mu, r)=\overline{1}-\mu \geq \operatorname{SSC}_{\tau}\left(f^{-1}(\overline{1}-v), r\right)$.

From (3),

$$
\begin{aligned}
\overline{1}-\mu & \geq \operatorname{SSC}_{\tau}\left(f^{-1}(\overline{1}-v), r\right) \\
& \geq f^{-1}\left(I_{\eta}\left(C_{\eta}(\overline{1}-v, r), r\right)\right)
\end{aligned}
$$

It implies

$$
\begin{aligned}
\mu & \leq \overline{1}-f^{-1}\left(I_{\eta}\left(C_{\eta}(\overline{1}-v, r), r\right)\right) \\
& =f^{-1}\left(\overline{1}-I_{\eta}\left(C_{\eta}(\overline{1}-v, r), r\right)\right) \\
& \mu \leq f^{-1}\left(C_{\eta}\left(I_{\eta}(v, r), r\right)\right)
\end{aligned}
$$

Therefore a $r$-fpc set $v \in I^{Y}$, with $v \geq C_{\eta}\left(I_{\eta}(v, r), r\right) \quad \ni$ $f^{-1}\left(C_{\eta}\left(I_{\eta}(v, r), r\right)\right) \geq \mu$ exists.

$(4) \Rightarrow(1)$ : Take a $r$-fssc set $\omega \in I^{X}$ and $v=\overline{1}-f(\omega)$ and $\mu=\overline{1}-\omega \ni \mu$ is $r$-fsso. We obtain

$f^{-1}(v)=f^{-1}(\overline{1}-f(\omega))=\overline{1}-f^{-1}(f(\omega)) \leq \overline{1}-\omega=\mu$.

(4) implies, a $r$-fpc set $\rho \in I^{Y}$ with $v \geq \rho \ni f^{-1}(\rho) \geq$ $\mu=\overline{1}-\omega$ exists. Thus $\omega \geq \overline{1}-f^{-1}(\rho)=f^{-1}(\overline{1}-\rho)$. Hence

$$
f(\omega) \geq f\left(f^{-1}(\overline{1}-\rho)\right)=\overline{1}-\rho .
$$

On the other hand, since $v \leq \rho$,

$$
f(\omega)=\overline{1}-v \leq \overline{1}-\rho
$$

Hence from (2.4) and (2.5), we have $f(\omega)=\overline{1}-\rho$. (i.e) $f(\omega)$ is $r$-fpo. Thus (1).

Theorem 2.9. For a bijective mapping $f:(X, \tau) \rightarrow(Y, \eta)$, 
(1) $f$ is a FcS-PC iff $f^{-1}\left(I_{\eta}\left(C_{\eta}(v, r), r\right)\right) \leq \operatorname{SSC}_{\tau}\left(f^{-1}(v)\right.$, $r), \forall v \in I^{Y}$ and $r \in I_{0}$.

(2) $f$ is a FcS-PC iff $f$ is FcS-PO.

Proof. (1) $\Rightarrow$ : Let $f$ be FcS-PC. Theorem 2.8(2), implies $\forall \mu \in I^{X} \& r \in I_{0}$,

$$
f\left(\operatorname{SSC}_{\tau}(\mu, r)\right) \geq I_{\eta}\left(C_{\eta}(f(\mu), r), r\right) .
$$

$\forall v \in I^{Y}, r \in I_{0}$. Take $\mu=f^{-1}(v)$, and the fact $f$ is surjective implies, $f\left(f^{-1}(v)\right)=v$. Thus,

$$
\begin{aligned}
f\left(\operatorname{SSC}_{\tau}\left(f^{-1}(v), r\right)\right) & \geq I_{\eta}\left(C_{\eta}\left(f\left(f^{-1}(v)\right), r\right), r\right) \\
& =I_{\eta}\left(C_{\eta}(v, r), r\right) .
\end{aligned}
$$

It implies

$$
\begin{aligned}
\operatorname{SSC}_{\tau}\left(f^{-1}(v), r\right) & =f^{-1}\left(f\left(\operatorname{SSC}_{\tau}\left(f^{-1}(v), r\right)\right)\right) \\
& \geq f^{-1}\left(I_{\eta}\left(C_{\eta}(v, r), r\right)\right) .
\end{aligned}
$$

$(\Leftarrow)$ Take $v=f(\mu)$. Since $f$ is $1-1$,

$$
\begin{aligned}
f^{-1}\left(I_{\eta}\left(C_{\eta}(f(\mu), r), r\right)\right) & \leq \operatorname{SSC}_{\tau}\left(f^{-1}(f(\mu)), r\right) \\
& =\operatorname{SSC}_{\tau}(\mu, r) .
\end{aligned}
$$

Since $f$ is surjective,

$$
I_{\eta}\left(C_{\eta}(f(\mu), r), r\right) \leq f\left(\operatorname{SSC}_{\tau}(\mu, r)\right) .
$$

$\Rightarrow f$ is $\mathrm{FcS}-\mathrm{PC}$.

(2) It is easily proved from :

$f^{-1}\left(I_{\eta}\left(C_{\eta}(v, r), r\right)\right) \leq S S C_{\tau}\left(f^{-1}(v), r\right)$

$\Leftrightarrow f^{-1}\left(\overline{1}-C_{\eta}\left(I_{\eta}(\overline{1}-v, r), r\right)\right) \leq \overline{1}-\operatorname{SSI}_{\tau}\left(\overline{1}-f^{-1}(v), r\right)$

$\Leftrightarrow \overline{1}-f^{-1}\left(C_{\eta}\left(I_{\eta}(\overline{1}-v, r), r\right)\right) \leq \overline{1}-\operatorname{SSI}_{\tau}\left(f^{-1}(\overline{1}-v), r\right)$

$\Leftrightarrow f^{-1}\left(C_{\eta}\left(I_{\eta}(\overline{1}-v, r), r\right)\right) \geq \operatorname{SSI}_{\tau}\left(f^{-1}(\overline{1}-v), r\right)$

$\Leftrightarrow$ Therefore $f$ is FcS-PO.

Theorem 2.10. (1) If $f: X \rightarrow Y$ is FS-IO and $g: Y \rightarrow Z$ is $F c S-P O$, then $g \circ f: X \rightarrow Z$ is FcS-PO.

(2) If $f: X \rightarrow Y$ is FS-IC and $g: Y \rightarrow Z$ is FcS-PC, then $g \circ f: X \rightarrow Z$ is FcS-PC.

(3) If $f: X \rightarrow Y$ is FcS-PO and $g: Y \rightarrow Z$ is FIPC, then $g \circ f: X \rightarrow Z$ is FcS-PO.

(4) If $f: X \rightarrow Y$ is FcS-PC and $g: Y \rightarrow Z$ is FIPO, then $g \circ f: X \rightarrow Z$ is FcS-PC.

(5) If $f: X \rightarrow Y$ is FcS-PCts and $g: Y \rightarrow Z$ is FsSCts, then $g \circ f: X \rightarrow Z$ is FcPCts.

(6) If $f: X \rightarrow Y$ is FcS-PCts and $g: Y \rightarrow Z$ is FS-ICts, then $g \circ f: X \rightarrow Z$ is FcS-PCts.

Proof. (1) Let $\lambda$ be $r$-fsso set in $X$. As $f$ is FS-IO, $f(\lambda)$ is $r$-fsso set of $Y$. Also since $g: Y \rightarrow Z$ is FcS-PO, $g(f(\lambda))$ is $r$-fpc in $Z$. (i.e) $(g \circ f)(\lambda)=g(f(\lambda))$ is $r$-fpc in $Z$. Thus $g \circ f$ is $\mathrm{FcS}-\mathrm{PO}$.

(2) - (6): Similar proof can be given.

\section{Conclusion}

In this article, fuzzy contra S-pre-continuous, fuzzy contra S-preopen, fuzzy contra S-preclosed mappings and fuzzy contra S-pre-homeomorphism with properties have been introduced and studied in Šstak's sense. Also a comparison between these new types of functions have been established by giving examples.

\section{References}

[1] S. E. Abbas and M. Azab Abd-alla, On fuzzy $S$-irresolute Continuous Mappings, The Journal of Fuzzy Mathematics, 12(4) (2004), 905-920.

[2] S. E. Abbas and I. M. Taha, Weaker forms of fuzzy contra continuity in fuzzy topological spaces, The Journal of Fuzzy Mathematics, 20(4) (2012), 857-876.

[3] Bai Shi Zhong, Fuzzy strongly semiopen and fuzzy strong semicontinuity, Fuzzy Sets and Systems, 52(1992), 345351.

[4] Bai Shi Zhong, Fuzzy S-irresolute mappings, J. of fuzzy math., 4(2) (1996), 397-411.

[5] C. L. Chang, Fuzzy topological spaces, J. Math. Anal. Appl., 24(1968), 182-189.

[6] K. C. Chattopadhyay, R. N. Hazra and S. K. Samanta, Gradation of openness, Fuzzy Sets and Systems, 49(2) (1992), 237-242.

[7] K. C. Chattopadhyay and S. K. Samanta, Fuzzy topology: fuzzy closure operator, fuzzy compactness and fuzzy connectedness, Fuzzy Sets and Systems, 54(2) (1993), 207-212.

[8] M. Caldas, G. Navalagi, R. Saraf, On some functions concerning fuzzy pg-closed sets, Proyecciones, Universidad Catolica del Norte Antofagasta., 25(3) (2006), 261-271.

[9] M. K. EI Gayyar, E. E. Kerre and A. A. Ramadan, Almost compactness and near compactness in Smooth topological spaces, Fuzzy Sets and Systems, 62(1994), 193-202.

[10] R. N. Hazra, S. K. Samanta and K. C. Chattopadhyay, Fuzzy topology redefined, Fuzzy Sets and Systems, 4(1992), 79-82.

[11] U. Höhle, Upper semicontinuous fuzzy sets and applications, J. Math. Anall. Apll., 78(1980), 659-673.

${ }^{[12]}$ U. Höhle and A. P. Šostak, A general theory of fuzzy topological spaces, Fuzzy sets and systems, 73(1995), 131-149.

[13] U. Höhle and A. P. Šostak, Axiomatic Foundations of Fixed-Basis fuzzy topology, The Handbooks of Fuzzy sets series, Volume 3, Kluwer Academic Publishers, Dordrecht (Chapter 3)(1999), 123-272.

[14] T. Kubiak, On fuzzy topologies, Ph.D. Thesis, A. Mickiewicz, Poznan, (1985).

[15] T. Kubiak and A. P. Šstak, Lower set-valued fuzzy topologies, Quaestions Math., 20(3) (1997), 423-429.

[16] Y. C. Kim, A. A. Ramadam and S. E. Abbas, Weaker forms of continuity in Šostak fuzzy topology, Indian J. Pure Appl. Math., 34(2) (2003), 311-333. 
[17] Y. C. Kim, A. A. Ramadam and S. E. Abbas, $r$-fuzzy strongly preopen sets in fuzzy topological spaces, Mathematicki vesnik., 55(2) (2003), 1-13.

${ }^{[18]}$ P. M. Pu and Y. M. Liu, Fuzzy topology. I. Neighborhood structure of a fuzzy point and Moore-Smith Convergence*., J. Math. Anal., 76 (1980), 571-599.

[19] A. A. Ramadan, Smooth topological spaces, Fuzzy Sets and Systems 48 (1992), 371-375.

[20] S. E. Rodabaugh, Categorical foundations of variable basis topology, in U. Höhle, S. E. Rodabaugh(Eds)), The Handbooks of fuzzy sets series, 3, Fuzzy sets : Logic, topology and Measure Theory, Kluwer Academic Publishers, Dordrecht (1991).

[21] A. P. Šostak, On a fuzzy topological structure, Rend. Circ. Matem. Palermo Ser. II 11(1985), 89-103.

[22] A. P. Šostak, Two decades of fuzzy topology : Basic ideas, Notion and results, Russian Math. Surveys, 44(6) (1989), 125-186.

[23] A. P. Šostak, On some modifications of fuzzy topologies, Mathematicki vesnik, 41(1989), 20-37.

[24] A. P. Šostak, On the neighbourhood structure of fuzzy topologies, Zb. Radova Univ. Nisu, 4 (1999), 7-14.

${ }^{[25]}$ S. Singh S, Generalization of certain fuzzy topological concepts, Ph.D. Dissertation (1999), R. D. University, Jabalpur (M. P.).

[26] B.Vijayalakshmi, M. Angayarkanni, P. Dhanasekaran and A. Vadivel, Fuzzy contra S-irresolute continuous mappings in Sostak's fuzzy topological spaces, (submitted).

[27] B.Vijayalakshmi, P. Dhanasekaran, M. Angayarkanni and A. Vadivel, Fuzzy contra Semi-S-irresolute continuous mappings in Ŝstak's fuzzy topological spaces, (submitted).

[28] M. S. Ying, A new approach for fuzzy topology(I), Fuzzy sets and systems, 39(1991), 303-321.

[29] L. A. Zadeh, Fuzzy sets, Inform. Control, 8(1965), 338353. 